\title{
A NOTE ON STOCHASTIC BOUNDS FOR QUEUEING NETWORKS
}

\author{
PANTELIS TSOUCAS* AND \\ JEAN WALRAND,* University of California, Berkeley
}

\begin{abstract}
Recently, Massey [1] proved that the vector of queue lengths of some queueing networks is stochastically dominated at any given time by that of a corresponding system of parallel $M / M / 1$ queues. This result is interesting, even though the bounds are generally quite conservative, in that the transient behavior of independent parallel $M / M / 1$ queues is considerably easier to analyze than that of a network.

This note provides an alternative proof of a generalized form of that result.
\end{abstract}

\section{Notation and basic lemma}

For $a:=\left(a^{1}, \cdots, a^{d}\right)$ and $b \in R^{d}(d \geqq 1), a \geqq b$ will indicate that $a^{i} \geqq b^{i}$ for $i=$ $1, \cdots, d$. Let $X, Y$ be two $R^{d}$-valued random variables. One writes

$$
\begin{aligned}
& X \geqq Y \quad \text { if } \quad \operatorname{Pr}\{X \geqq a\} \geqq \operatorname{Pr}\{Y \geqq a\}, \quad \text { for all } a \in R^{d}, \\
& X \underset{\mathrm{s}}{\overline{\mathrm{s}}} \quad \text { if } \quad X \geqq Y \quad \text { and } \quad Y \geqq X .
\end{aligned}
$$

(Thus $X \geqq Y$ indicates that $X$ and $Y$ have the same probability distribution function.)

For $x, y \in R, x \wedge y:=\min \{x, y\}$ and $x^{+}=\max \{x, 0\}$. Let $Z_{+}=\{0,1,2, \cdots\}$.

Lemma. Let $X, Y, M, N$ be $Z_{+}^{2}$-valued random variables such that $X \geqq Y,\{X, Y\}$ and $M$ are independent, and given $Y$,

$$
N=\left(N^{1}, N^{2}\right) \underset{\mathrm{s}}{=}\left(N^{2}, N^{1}\right), \quad \text { and } \quad\left(-M^{1}, M^{2}\right) \geqq\left(-N^{1}, N^{2}\right) \text {. }
$$

Then

$$
\left(\left(X^{-1}-M^{1}\right)^{+}, X^{2}+M^{2}\right) \underset{\mathrm{s}}{\geqq}=\left(\left(Y^{1}-N^{1}\right)^{+}, Y^{2}+N^{1} \wedge Y^{1}\right) .
$$

Received 7 June 1984; revision received 30 August 1984.

* Postal address: Department of Electrical Engineering and Computer Sciences and Electronics Research Laboratory, University of California, Berkeley, CA 94720, USA.

Research supported in part by NSF Grant No. ECS-8205428. 
Proof. By conditioning on $M$ one finds that

$$
\left(\left(X^{1}-M^{1}\right)^{+}, X^{2}+M^{2}\right) \underset{\mathrm{s}}{\geqq}\left(\left(Y^{1}-M^{1}\right)^{+}, Y^{2}+M^{2}\right)=: V .
$$

To show $V \geqq W$ one must prove that for all $m^{1}, m^{2} \in Z_{+}$,

$$
\operatorname{Pr}\left\{V^{1} \geqq m^{1}, V^{2} \geqq m^{2}\right\} \geqq \operatorname{Pr}\left\{W^{1} \geqq m^{1}, W^{2} \geqq m^{2}\right\} .
$$

For $m^{1}=0$, (1) reads $V^{2} \geqq W^{2}$ and follows from

$$
Y^{2}+M^{2} \underset{\mathrm{s}}{\geqq} Y^{2}+N^{2}=Y_{\mathrm{s}}^{2}+N^{1} \underset{\mathrm{s}}{\geqq} Y^{2}+N^{1} \wedge Y^{1} .
$$

For $m^{1}>0,(1)$ is implied by the following inequality:

$$
\left(Y^{1}-M^{1}, Y^{2}+M^{2}\right) \underset{\mathrm{s}}{\geqq}\left(Y^{1}-N^{1}, Y^{2}+N^{1}\right) .
$$

To see why (2) holds, observe that

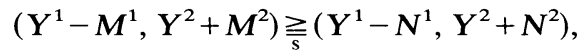

so that it suffices to show that

$$
\left(Y^{1}-N^{1}, Y^{2}+N^{2}\right) \underset{\mathrm{s}}{\gtrless}\left(Y^{1}-N^{1}, Y^{2}+N^{1}\right) .
$$

By conditioning on $Y$ it remains only to prove that for all $m^{1}, m^{2} \in Z_{+}$

$$
\operatorname{Pr}\left\{N^{1} \leqq m^{1}, N^{2} \geqq m^{2}\right\} \geqq \operatorname{Pr}\left\{N^{1} \leqq m^{1}, N^{1} \geqq m^{2}\right\} .
$$

But this last inequality is immediate from $\left(N^{1}, N^{2}\right)=\left(N^{2}, N^{1}\right)$.

\section{Stochastic networks}

Let $\left\{x_{t}, y_{t}, t \geqq 0\right\}$ be two $Z_{+}^{d}$-valued processes corresponding to the vectors of queue lengths in two networks of $d$ queues. Alternatively, each $i \in\{1, \cdots, d\}$ could be some $(k, c) \in\{1, \cdots, K\} \times\{1, \cdots, C\}$ where $c$ would indicate a customer class and $k$ a node number. Other variations are possible.

Assume that those processes admit the following representation. For $t \geqq 0$ and $i \in\{1, \cdots, d\}$,

$$
\begin{aligned}
& d x_{t}^{i}=-\sum_{j=0}^{d} 1\left\{x_{t-}^{i}>0\right\} d S_{t}^{i j}+\sum_{j=0}^{d} d A_{t}^{i i} \\
& d y_{t}^{i}=-\sum_{j=0}^{d} 1\left\{y_{t-}^{i}>0\right\} d R_{t}^{i j}+\sum_{j=0}^{d} 1\left\{y_{t-}^{j}>0\right\} d R_{t}^{i j} .
\end{aligned}
$$

In these expressions, the differential equations are in the Lebesgue-Stieltjes sense, $y_{\mathrm{t}}^{0} \equiv 1$, and the processes $R^{i j}, A^{i j}, S^{i j}$ are point processes with the following properties. The processes $S_{i j}, A^{i j}, \tilde{A}^{i j}$ (see below) are Poisson processes; $\left\{S^{i j}, \tilde{A}^{i j}\right\}, A^{i j}$ for $0 \leqq i, j \leqq d$ are independent; for every $(i, j), A^{i j}$ and $\tilde{A}^{i j}$ have the same rate; almost surely one has

$$
\Delta S_{t}^{i j}:=S_{t}^{i j}-S_{t-}^{i j} \leqq \Delta R_{t}^{i j} \leqq \Delta \tilde{A}_{t}^{i j} \text { for } t \geqq 0,0 \leqq i, j \leqq d .
$$

Thus $x_{t}$ corresponds to a system of parallel $M / M / 1$ queues while $y_{t}$ corresponds to a network of interconnected queues and need not be Markov. The routing and the service rates in $y_{t}$ may depend on the 'state' of the complete network; the basic assumption is 
that the service rate from queue $i$ to queue $j$ is bounded from above and from below when the queue is not empty.

The following proposition extends a result of Massey [1].

Proposition. Assume that $x_{0} \geqq y_{0}$. Then $X_{\mathrm{T}} \geqq y_{\mathrm{s}}$ for all $t \geqq 0$.

Proof. Let $T_{1}, T_{2}, \cdots$ be the successive jump times of $\sum_{i j}\left(S_{t}^{i j}+A_{t}^{i j}+\tilde{A}_{t}^{i j}\right)$ and define $X_{n}=x_{T_{n}}, Y_{n}=y_{T_{n}}$ for $n \geqq 1$.

It suffices to show that given $\left\{T_{m}, m \geqq 1\right\}, X_{n} \geqq Y_{n}$. Assume that this is true for $n$.

Notice that given

$$
\Psi:=\left\{X_{n}, Y_{n}, T_{m}, m \geqq 1, \Delta A_{T_{n+1}}^{i j}+\Delta \tilde{A}_{T_{n+1}}^{i j}=1\right\},
$$

the following random variables

$$
\begin{aligned}
& N^{1}=\Delta R_{T_{n+1}}^{i j}, M^{1}=\Delta S_{T_{n+1}}^{i j}, M^{2}=\Delta A_{T_{n+1}}^{i j}, \\
& X^{1}=X_{n}^{i}, X^{2}=X_{n}^{i}, Y^{1}=Y_{n}^{i}, Y^{2}=Y_{n}^{i}
\end{aligned}
$$

satisfy the conditions of the lemma with $N^{2}$ being an i.i.d. copy of $N^{1}$ (given $\Psi$ ). Hence, given $\Psi, X_{n+1} \gtreqless Y_{n+1}$, and this concludes the proof.

For instance, if network $Y$ consists of $d$ queues with exogenous arrival rates $\lambda_{i}$, service rates in $\left[a_{i}, b_{i}\right]$ in queue $i$ when it is non-empty, and routing probabilities $r_{i j}$, then $y_{\mathrm{t}}$ is stochastically dominated by the vector of queue lengths of $d$ parallel queues with arrival rates $\lambda_{i}+\sum_{i} b_{i} r_{i i}$ and service rates $a_{i}$. The proposition shows that this result holds in a more general context.

\section{Remarks.}

(1) The result extends to deterministic, and therefore to arbitrary arrivals, by applying the argument to the processes between arrival times.

(2) The idea of considering the Markov chain $\left\{Y_{n}\right\}$ shows that if a network of $M / M / s$ queues and arbitrary arrivals is started with stochastically more customers, then that ordering is preserved at all times.

(3) It can be shown that it is not possible to construct $x_{t}$ and $y_{t}$ on the same probability space in such a way that $\operatorname{Pr}\left\{x_{t} \geqq y_{t}\right.$, for all $\left.t \geqq 0\right\}=1$. That is, the domination is not pathwise. (See [2].)

\section{Acknowledgements}

This note benefited from discussions with Professor P. Varaiya and with Dr W. Massey.

\section{References}

[1] MASSEY, W. (1984) Open networks of queues: their algebraic structure and estimating their transient behavior. Adv. Appl. Prob. 16, 176-201.

[2] MAsSEY, W. (1984) An operator-analytic approach to the Jackson network. J. Appl. Prob. 21, 379-393. 